\title{
Transient embolic occlusion of the middle cerebral and internal carotid arteries in cerebral apoplexy
}

\author{
C. FIESCHI AND L. BOZZAO
}

From the Department of Neurology and Psychiatry, University of Genoa School of Medicine, Genoa

Published records of carotid angiography in cases of hemispheric infarctions report different frequencies of occlusion of the cerebral arteries (Riishede, 1957; Bull, Marshall, and Shaw, 1960; Gurdjian, Lindner, Hardy, and Webster, 1960; Gurdjian, Lindner, Hardy, and Thomas, 1961; Bauer, Sheehan, Wechsler, and Meyer, 1962; Silverstein, 1962; Newton, Adams, and Wylie, 1964; Silverstein, 1965).

Several factors may explain such differences: in the first place, the criteria of admission and selection of the patients submitted to angiography.

In a previous paper (Fieschi, 1965) the time interval between the onset of symptoms and the angiographic study was taken into account. It was noted that the earlier the angiography was effected, the higher the frequency of occlusion; this difference was especially pronounced in the occlusion of the intracranial arteries, such as the middle cerebral artery (MCA). A similar remark has been made by Luessenhop (1959), Jacobsen and Skinhøj (1959), and Torvik and Jörgensen (1964). In fact, the frequency of middle cerebral artery occlusion ranges from 25 to $30 \%$ of cases of brain softening studied within the first few days of the stroke by Riishede (1957), McDowell, Schick, Frederick, and Dunbar (1959), Frantzen, Harvald, and Hangsted (1959), and Fieschi (1965), to 4 to $8 \%$ of the cases studied at a later period after the acute phase (Gurdjian et al., 1960, 1961; Bauer et al., 1962). The progressively decreasing frequency of occlusion of large intracranial arteries was attributed to a higher and earlier death rate and/or to a true disappearance of the defect of canalization. This in turn may be explained by a lysis of a recent thrombus or embolus or by a haemodynamic mechanism - that is, regional changes of the peripheral resistances with 'intracerebral steal' (Fazio, 1968).

Dalal, Shah, Aiyar, and Kikani (1968) state that intracranial occlusions are much more frequent in cerebral embolism $(65 \%$ vs. $22 \%$ extracranial lesions) than in cerebral thrombosis $(20 \%$ intracranial vs. $39 \%$ extracranial lesions). They further note that spontaneous clot lysis is common in cerebral embolism, while it is not encountered in $\frac{\bar{\Phi}}{\widehat{D}}$ cerebral thrombosis.

Independently of its interpretation, the phenom- के enon of a transient occlusion is a quite important $\rightarrow$ one, as it would considerably reduce the number of brain infarctions unexplained by an arterial $\vec{\omega}$ occlusion.

In the past four years, we have performed the angiographic test on patients with cerebral vascular? injuries as soon as possible after the stroke.

In the present paper we report the findings $\bar{f} \omega$ such a study, showing that a transient occlusion of $\mathrm{w}_{\mathrm{W}}^{\mathrm{u}}$ the MCA or even of the internal carotid arte disappearing a few days after the stroke is a less rare occurrence than previously suspected.

\section{CASE MATERIAL}

Our study involved 100 patients hospitalized in the $\overrightarrow{0}$ Department of Mental and Nervous Diseases of to University of Genoa during the period of October 1963 to May 1968. Criteria of admission to the study were: (1) age $<75$ years; (2) clinical evidence of a major cerebral vascular accident in a cerebral hemisphere $\bar{\partial}$ within the previous 24 hours; (3) absence of clinical signs of gross intracranial hypertension with pressure cones $\mathbb{D}$ and secondary brain-stem damege; (4) vegetative and cardiorespiratory state judged as not contraindicating $\overrightarrow{\overline{0}}$ a cerebral angiography. Once admitted to the study, the 3 patients were submitted in the subsequent hours to routine neurological, laboratory, and general investigation including CSF examination, EEG, and ECG.

Because of rapid deterioration of their clinical condition or of other contraindication such as high blood? glucose or nitrogen levels, or recent myocardial infarc- $\frac{0}{3}$ tions, four patients were not submitted to the angiograph study. In the remaining 96 cases the angiographic test $\mathrm{O}$ took place within three days of the stroke.

Only the carotid system ipsilateral to the involved 0 hemisphere was examined in the acute phase, while $a_{\supset}$ complete angiographic study was effected on most of the patients in the subsequent stages.

Angiography was performed by directly canalizing the $\mathrm{N}$ common carotid artery in the neck; however, in the last ${ }^{\circ} \mathrm{N}$ three years, angiographic studies on patients with $\bigcirc$ involvement of the right hemisphere have been effected $\mathcal{W}$ by means of retrograde brachial angiography. 


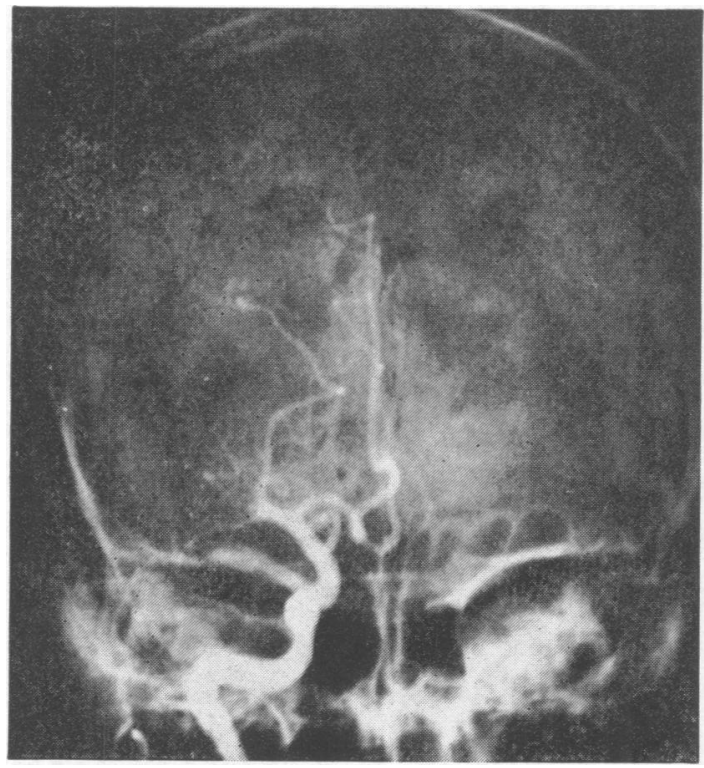

FIG. 1.

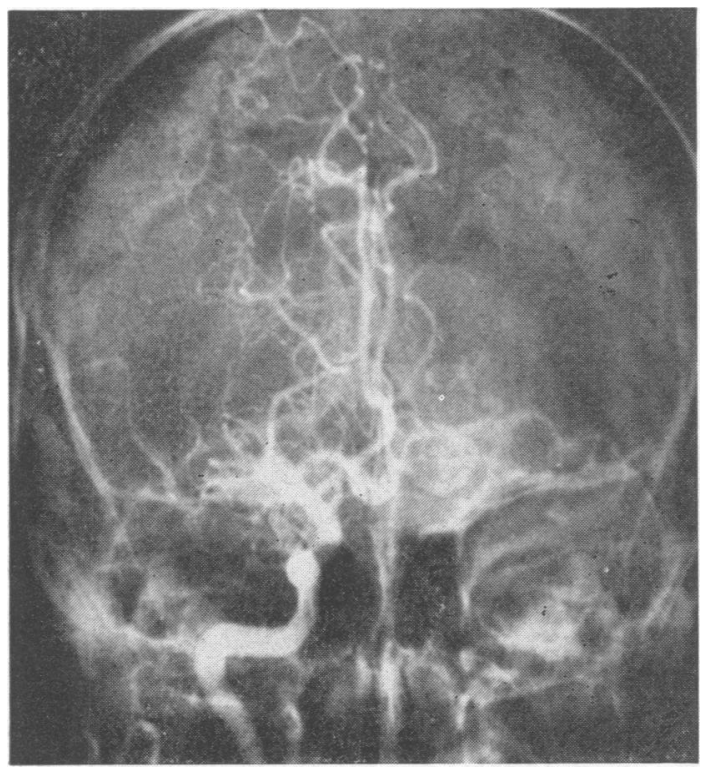

FIG. 2 .

FIGS 1 to 3. Case 1. Occlusion of the MCA two days after the stroke (Fig. 1). The occlusion seems incomplete, as anterior temporal branches are filled on a subsequent film (Fig. 2). The lateral seriography clearly showed collateral filling of cortical branches from the $A C A$ and $P C A$. Eight days later, disobliteration of the MCA (Fig. 3).

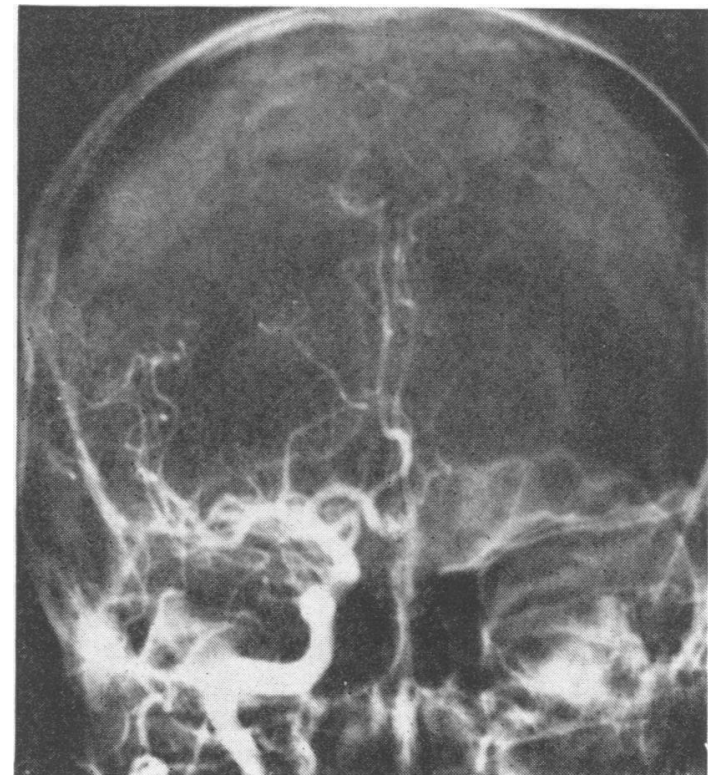

FIG. 3.

\section{RESULTS}

The present report concerns the 96 patients with acute hemispheric vascular lesions on whom the angiography was effected within three days of the stroke. A cerebral haemorrhage was diagnosed in 10 of these patients as a result of clinical, angiographic and/or post-mortem examination.

Of the 86 patients with cerebral ischaemic injuries, $49(57 \%)$ were found to have a complete arterial occlusion in the neck or in the intracranial part of the carotid artery and its branches ipsilateral to the involved hemisphere. In the remaining 37 patients the cerebral angiography showed localized or diffuse arteriosclerosis of more or less accentuated nature, without arterial occlusion.

In 22 of the patients with occlusive lesions the occlusion was found to be at the level of the internal carotid artery (ICA) in the neck (16 cases) or in the distal segment (intracavernous or supraclinoid: six cases). In 25 cases the middle cerebral artery (MCA) or one of its major branches was occluded, while in two cases the arterior cerebral artery (ACA) was involved.

The occlusions were observed at repeated-at least two-injections on several films and were accompanied by the development of various degrees of collateral circulation.

Angiographic or necropsy controls were obtained in 17 patients. The arterial occlusion was confirmed in seven cases (three with angiographic and four 
with necropsy control). In 10 of them (seven with angiographic and three with necropsy control) the vessels were patent.

The apparent 'disobliteration' of the occlusion was noted in eight cases of MCA occlusion (Figs 1-5), in two cases of distal occlusion of the ICA (Figs 6-8), and in no case of ACA occlusion.

\section{DISCUSSION}

Two points of our study are worthy of comment. The first is the high frequency of occlusion of the MCA in the acute phase of the stroke: in our case material 25 out of 86 patients examined within three days of the stroke, or $29 \%$. The second one is the high frequency with which an occlusion of the ICA was found at the level of the distal segment: six cases, or 7\% of the case record (Figs 6-8).

The high frequency of occlusion of the MCA in the present material is similar to that reported by previous authors who performed the angiographic study in the acute stage of the stroke.

This high incidence may partly be explained by the fact that MCA occlusion usually causes severe damage resulting in a higher death rate as compared with other ischaemic lesions. In Riishede's (1957) cases the death rate in patients with MCA occlusion was seven out of 10 , while in our case this was 10 out of 25 .

On the other hand it is possible that occlusions shown in the acute phase may recanalize in a later stage of the illness. In fact, previous publications report some cases where the angiographic or necropsy check-up effected a few weeks later shows the disappearance of the occlusion of a MCA observed in the acute phase (Ecker, 1945, one case; Lehrer, 1958, one case; Jacobsen and Skinhøj, 1959, two cases; Gannon and Chait, 1962, one case; Meyer, Gilroy, Barnhart, and Johnson, 1963, two cases; Dalal, Shah, and Aiyar, 1965, eight cases; Zatz, Iannone, Eckman, and Hecker, 1965, four cases). We are now adding seven cases in which a MCA occlusion diagnosed in the first or second day after the stroke had disappeared at the time of a later examination.

In six out of 22 cases with ICA occlusion the defect of canalization was located at the intracranial level. Occlusions of the ICA at the level of its distal segment (intracavernous or supraclinoid segments) are not very frequent in routine cerebral angiography performed at a later stage of cerebral vascular accident (Luessenhop, 1959). In addition to the cases with MCA and ACA occlusion, these patients raise to more than $38 \%$ the occlusions of intracranial arteries in acute apoplexy.

The frequency of occlusion of the ICA at the level of the distal segment in the acute phase, upholds the supposition of Luessenhop that in some cases the occlusion may extend downward to the bifurcation of the internal carotid artery at the neck. This would

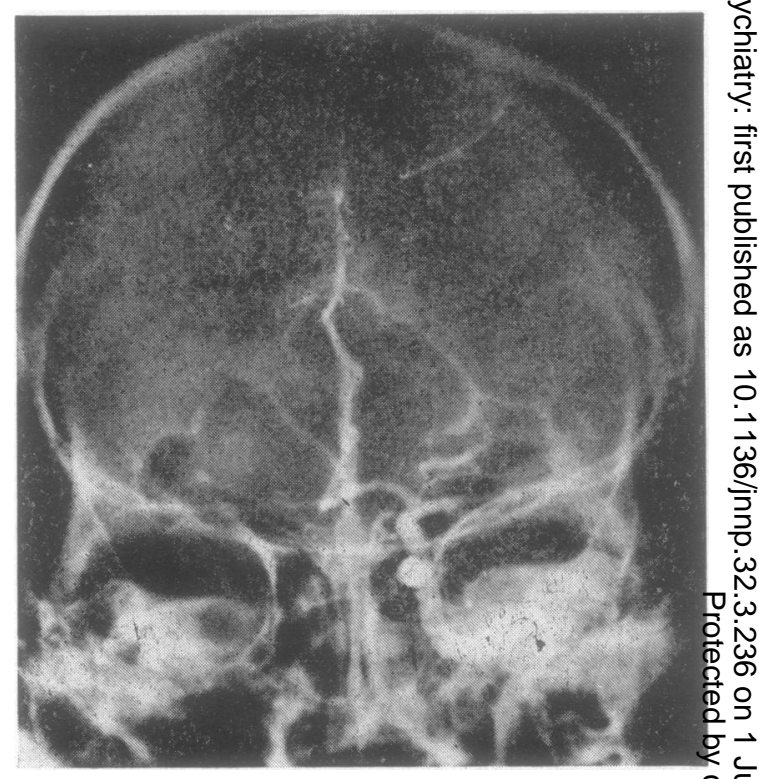

FIG. 4. Case 2. Occlusion of the MCA on the day of the stroke.

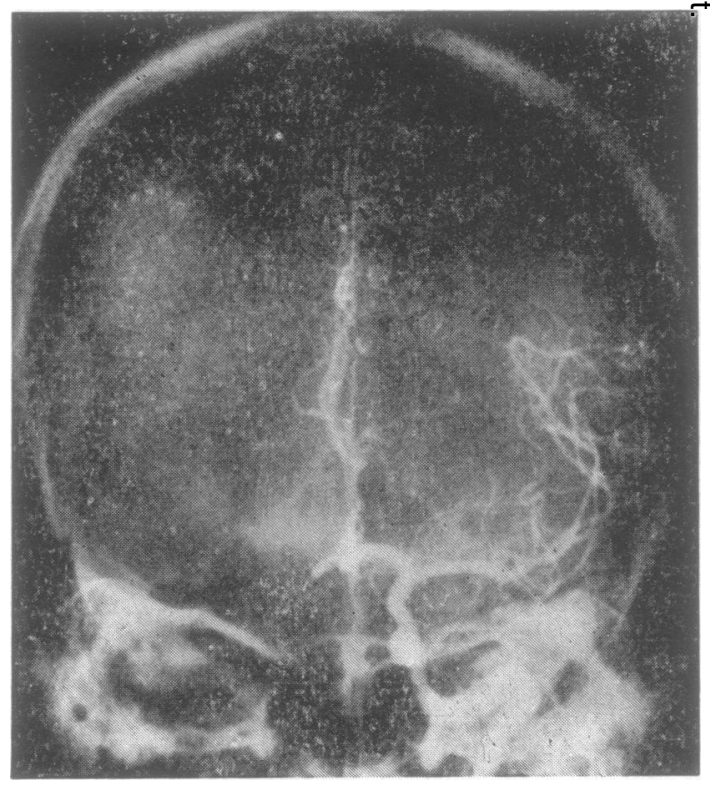

FIG. 5. Case 2. Disobliteration two weeks later. 


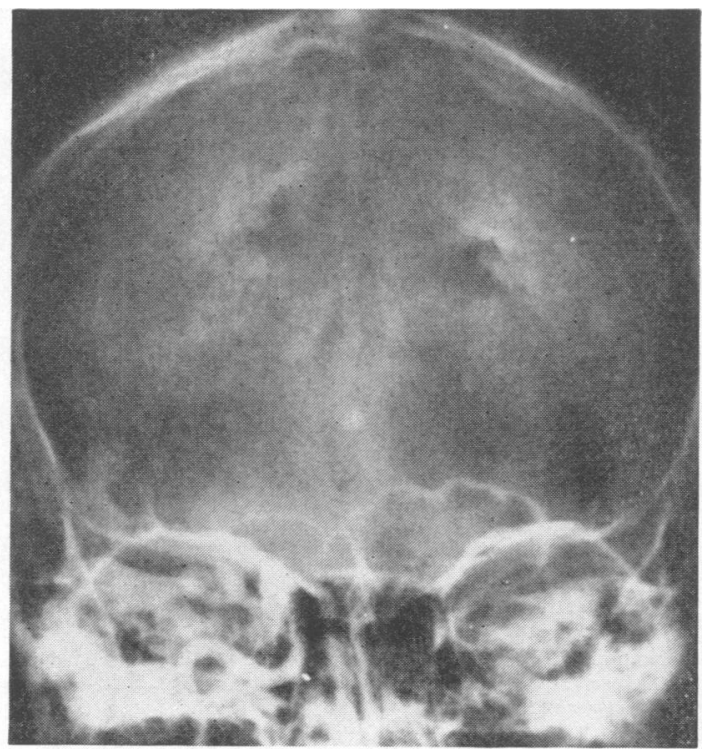

FIG. 6.

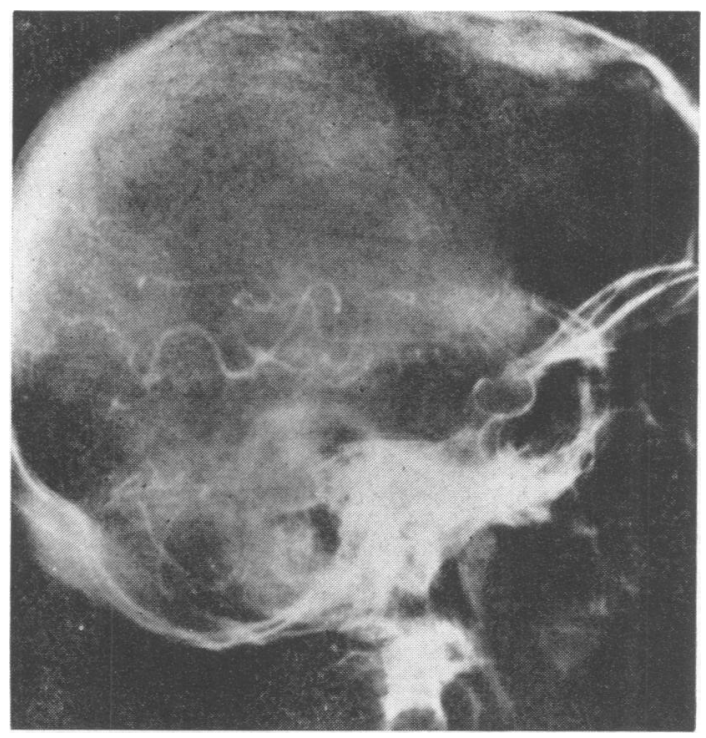

FIG. 7.

explain the higher frequency of occlusion at the origin of the ICA in most reports on less acute case material. However, in other cases the occlusion may disappear, probably due to the migration, fragmentation, or lysis of the embolus. In fact, in two of our six cases the occlusion had disappeared at an angiography effected two to three weeks later.

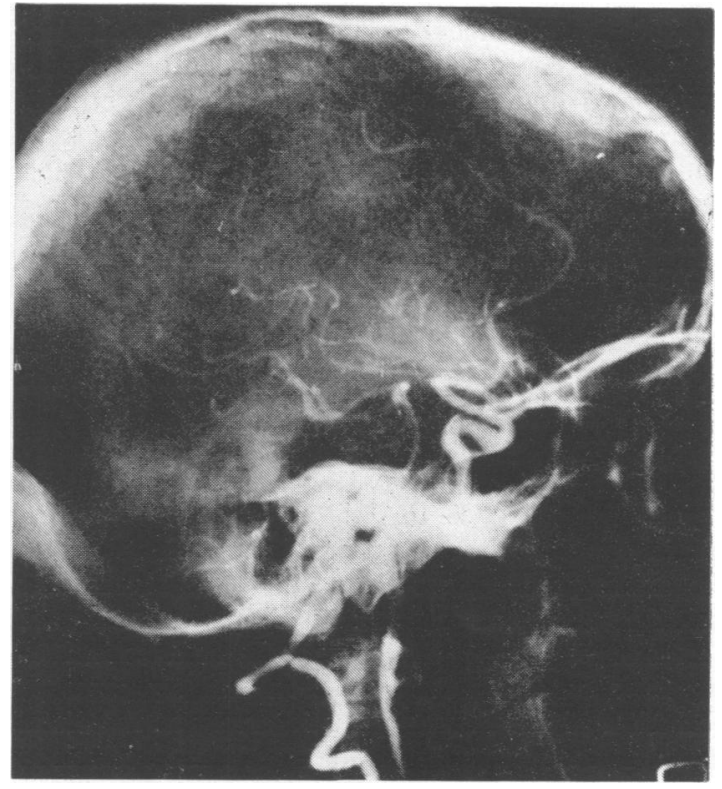

FIG. 8.

FIGS. 6 to 8. Case 3. Carotid occlusion at the level of the syphon. Angiography performed by direct puncture of the common carotid artery demonstrates a progressive narrowing of the ICA and only six seconds later the contrast medium reaches the syphon (Fig. 6). After four days a second angiography through the right brachial artery confirms an occlusion of the internal carotid artery at intracranial level. Branches of the $M C A$ are partly filled from the vertebro-basilar system, through cortical anastomoses with the posterior cerebral artery (Fig. 7). A third angiography performed 20 days later showed filling of the internal carotid artery and its intracranial branches, with disappearance of the occlusion seen twice in the first four days (Fig. 8).

The high frequency of occlusion of intracranial arteries in the first days after a major ischaemic accident in the cerebral hemispheres being clearly established, as well as the 'transient' nature of a number of these occlusions, the discussion concerns the incidence of this phenomenon in cases of brain infarcts and the nature of the occlusive process.

On the basis of our experience (10 cases of reestablished patency out of 17 cases of intracranial occlusion with angiographic and/or necropsy control) it appears legitimate to assume that such phenomenon of 'disappearance' of an arterial occlusion could be observed more frequently were the angiographies performed systematically in acute patients and followed by careful anatomical and/or radiological examination repeated at a later stage.

As far as the nature of the underlying process is 
concerned, the clinical records and the pathological findings in cases of transient occlusion are compatible with an embolus. However, not all the criteria required to make a diagnosis of embolism were fulfilled (see description of cases in Fieschi, 1965). Therefore, the genesis of these 'disappearing' occlusions is not proven, and a haemodynamic component cannot be entirely ruled out. Even less is known about the intimate nature of the process of lysis or fragmentation of a large embolus; a recent work of Dalal (1969) gives some clues to the solution of this problem.

\section{SUMMARY}

Angiographic studies performed in 86 patients during the acute phase of an ischaemic cerebral vascular lesion permitted us to note: (1) a high percentage of occlusion, especially of intracranial arteries including 25 cases of MCA occlusion and six cases of occlusion of the internal carotid artery at the distal level; (2) the recanalization of 10 of these occlusions in angiographic check-up repeated at a later date or at necropsy control.

Transient occlusion of the major cerebral arteries may thus play an important role in explaining the pathogenesis of brain infarcts.

\section{REFERENCES}

Bauer, R. B., Sheehan, S., Wechsler, N., and Meyer, J. S. (1962) Arteriographic study of sites, incidence, and treatment of arteriosclerotic cerebrovascular lesions. Neurology (Minneap.) 12, 698-711.

Bull, J. W. D., Marshall, J., and Shaw, D. A. (1960). Cerebra angiography in the diagnosis of the acute stroke. Lancet, 1 , $562-565$.

Dalal, P. M. (Personal communication.) J. clin. Path. (In press.)

- Shah, P. M., and Aiyar, R. R. (1965). Arteriographic study of cerebral embolism. Lancet, 2, 358-361.

,,--- and Kikani, B. J. (1968). Cerebrovascular diseases in West Central India. A report on angiographic findings from a prospective study. Brit. med. J., 3, 769-774.
Ecker, A. D. (1945). Spasm of the internal carotid artery. J. Neuro- Z surg., 2, 479-484. (Quoted by Jacobsen and Skinhøj, 1959.)

Fazio, C. (1968). Componenti emodinamiche nella patogenesi della apoplessia cerebrale, pp. 490-499, in Brain and Mind Problems, \& edited by R. Vizioli. Il Pensiero Scientifico: Roma.

Fieschi, C. (1965). Considerazioni sulla patogenesi dei rammollimenti cerebrali derivate dallo studio di casi acuti. Arch. Psicol. Neurol. Psichiat., 26, 143-173.

Frantzen, E., Harvald, B., and Hangsted, H. (1959). The arteriographic and electroencephalographic findings in cerebral apoplexy. Dan. med. Bull., 6, 12-19.

Gannon, W. E., and Chait, A. (1962). Occlusion of the middle cerebral artery with recanalization. Amer. J. Roentgenol., 88, 24-26.

Gurdjian, E. S., Lindner, D. W., Hardy, W. G., and Webster, J. E. (1960). Cerebrovascular disease. An analysis of 600 cases $\stackrel{\oplus}{\rightarrow}$ Neurology (Minneap.), 10, 372-380.

,,--- , and Thomas, L. M. (1961). Incidence of surgically $\frac{O}{C}$ treatable lesions in cases studied angiographically. Neurology $\overline{\bar{C}}$ (Minneap.), 11, 150-152.

Jacobsen, H. H., and Skinhøj, E. (1959). Occlusion of the middle $\overparen{\Phi}$ cerebral artery; an analysis of thirty-six arteriographed cases. Dan. Med. Bull., 6, 9-12.

Lehrer, G. M. (1SE3). Arteriographic demonstration of collateral circulation, in cerebrovascular disease. Neurology (Minneap.), 8, 27-32.

Luessenhop, A. J. (1959). Occlusive disease of the carotid artery. $\vec{\omega}$ Observations on the prognosis and surgical treatment. J.O Neurosurg., 16, 705-730.

McDowell, F. H., Schick, R. W., Frederick, W., and Dunbar, H. S. (1959). An arteriographic study of cerebrovascular disease.? Arch. Neurol. (Chic.), 1, 435-442.

Meyer, J. S., Gilroy, J., Barnhart, M. I., and Johnson, J. F. (1963) Therapeutic thrombolysis in cerebral thromboembolis Double-blind evaluation of intravenous plasmin therapy carotid and middle cerebral arterial occlusion. Neurology (Minneap.), 13, 927-937.

Newton, T. H., Adams, J. E., and Wylie, E. J. (1964). Arteriograp五y of cerebrovascular occlusive disease. New Engl. J. Med., 20 14-18.

Riishede, J. (1957). Cerebral apoplexy. An arteriographical and cliniêl study of 100 cases. Acta psychiat. scand., 32, (Suppl. no. 118), 1-163.

Silverstein, A. (1962). Angiography of ischaemic brain diseas. J. Mt Sinai Hosp., 29, 74-80.

(1965). Arteriography of stroke. II. Factors relating to the normal angiogram. Arch. Neurol. (Chic.), 13, 441-446.

Torvik, A., and Jörgensen, L. (1964). Thrombotic and embolic occlusions of the carotid arteries in an autopsy material. Part I. Prevalence, location and associated diseases. J. neur.l. Sci., 1, 24-39.

Zatz, L. M., Iannone, A. M., Eckman, P. B., and Hecker, S. P. $\stackrel{\mathbb{Q}}{\Omega}$ (1965). Observations concerning intracerebral vascular occlusion. Neurology (Minneap.), 15, 389-401. 